Supporting Information

\title{
Preparation and characterization of electrospun conductive Janus nanofibers with polyaniline
}

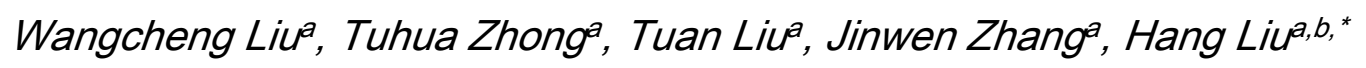

a Composite Materials and Engineering Center, Washington State University, Pullman,

WA 99164, USA

${ }^{\mathrm{b}}$ Apparel, Merchandising, Design and Textiles, Washington State University, Pullman, WA 99164, USA

Keywords: Multicomponent, side-by-side, electrospinning, polyaniline, conductive 
${ }^{*}$ Corresponding author.

E-mail address. hangliu@wsu.edu (H. Liu)

Table S1. Viscosities of polymer solutions at shearing rate of $1 \mathrm{rad} / \mathrm{s}$.

\begin{tabular}{|c|c|c|}
\hline Viscosity (mPa.s) & No PANI & With PANI-CSA (3 w/v) \\
\hline No PEO or PVP & $<2$ & $<2$ \\
\hline PEO $(3 \mathrm{w} / \mathrm{v})$ & $1530 \pm 35$ & $1647 \pm 19$ \\
\hline PVP $(10 \mathrm{w} / \mathrm{v})$ & $435 \pm 7$ & $811 \pm 6$ \\
\hline
\end{tabular}



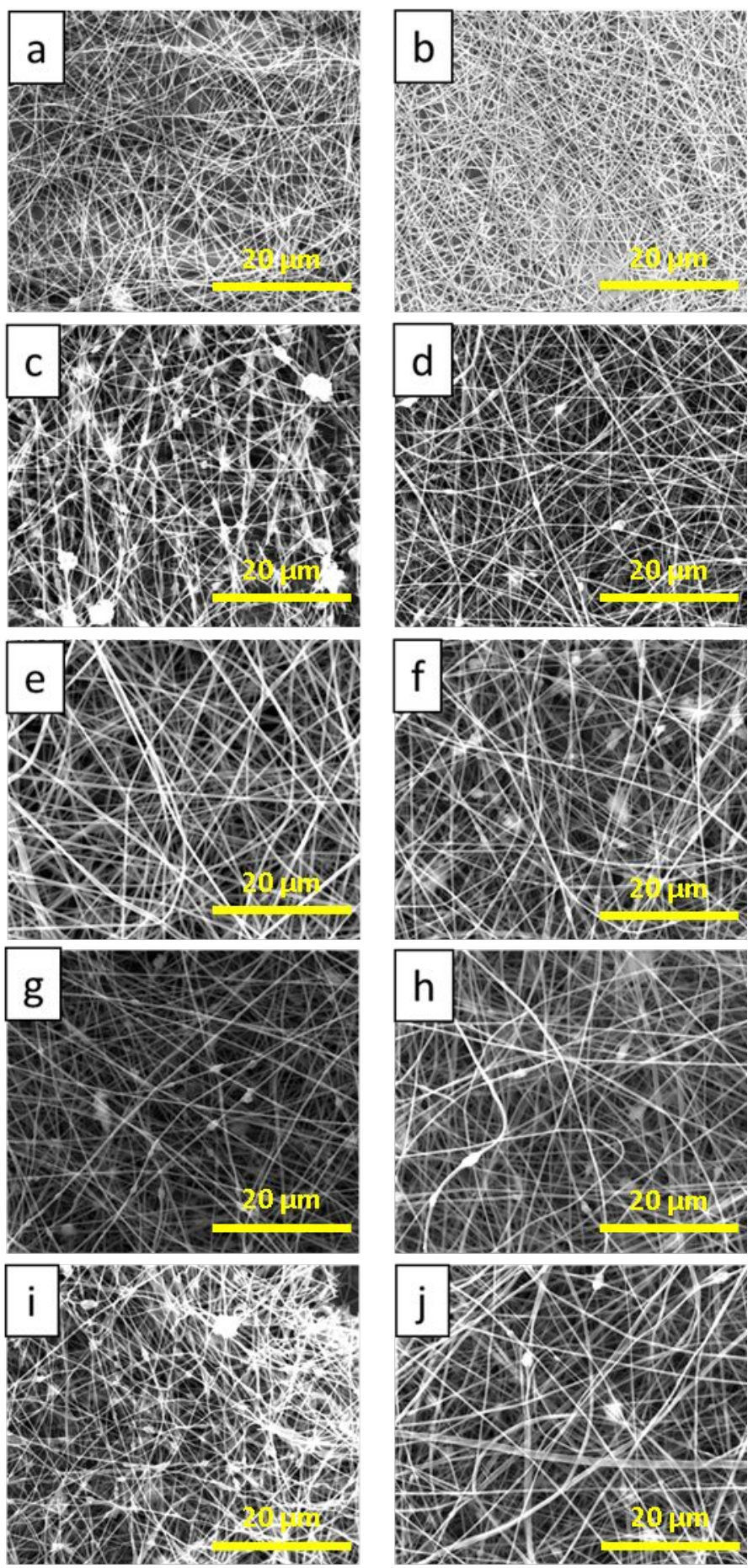

Figure S1. SEM images of electrospun nanofiber in low magnification (5000×). (a) PEO S; (b) PVP S; (c) PEO-PANI S; (d) PVP-PANI S; (e) PEO/PVP SBS; (f) PEO-PANI/PVP-PANI SBS; (g) PVP/PEO-PANI SBS; (h) PEO/PVP-PANI SBS; (i) PEO/PEO-PANI SBS and (j) PVP/PVPPANI SBS. 
Table S2. Nanofiber diameters and voltage used for preparing the fibers.

\begin{tabular}{|c|c|c|c|}
\hline \multicolumn{2}{|r|}{ Code } & Diameter (nm) & Spinning Voltage \\
\hline \multirow{4}{*}{ Ss } & PEO & $125 \pm 27$ & 8 \\
\hline & PVP & $159 \pm 56$ & 12 \\
\hline & PEO-PANI & $119 \pm 36$ & 8 \\
\hline & PVP-PANI & $142 \pm 61$ & 12 \\
\hline \multirow{6}{*}{ SBSs } & PEO/PVP & $317 \pm 68$ & 10 \\
\hline & $\begin{array}{c}\text { PEO-PANI/PVP- } \\
\text { PANI }\end{array}$ & $281 \pm 43$ & 10 \\
\hline & PVP/PEO-PANI & $265 \pm 44$ & 10 \\
\hline & PEO/PVP-PANI & $290 \pm 82$ & 10 \\
\hline & PEO/PEO-PANI & $181 \pm 46$ & 8 \\
\hline & $\begin{array}{l}\text { PVP/PVP-PANI } \\
\end{array}$ & $281 \pm 99$ & 12 \\
\hline
\end{tabular}

All electrospun nanofibers had average diameters between 120 and $320 \mathrm{~nm}$. The additional of PANI slightly reduced fiber diameters because of its conductive nature, which increased jet stretching during spinning. The diameters of SBS nanofibers were close to the sum of the S fibers spun from the two components in the SBS fibers. 

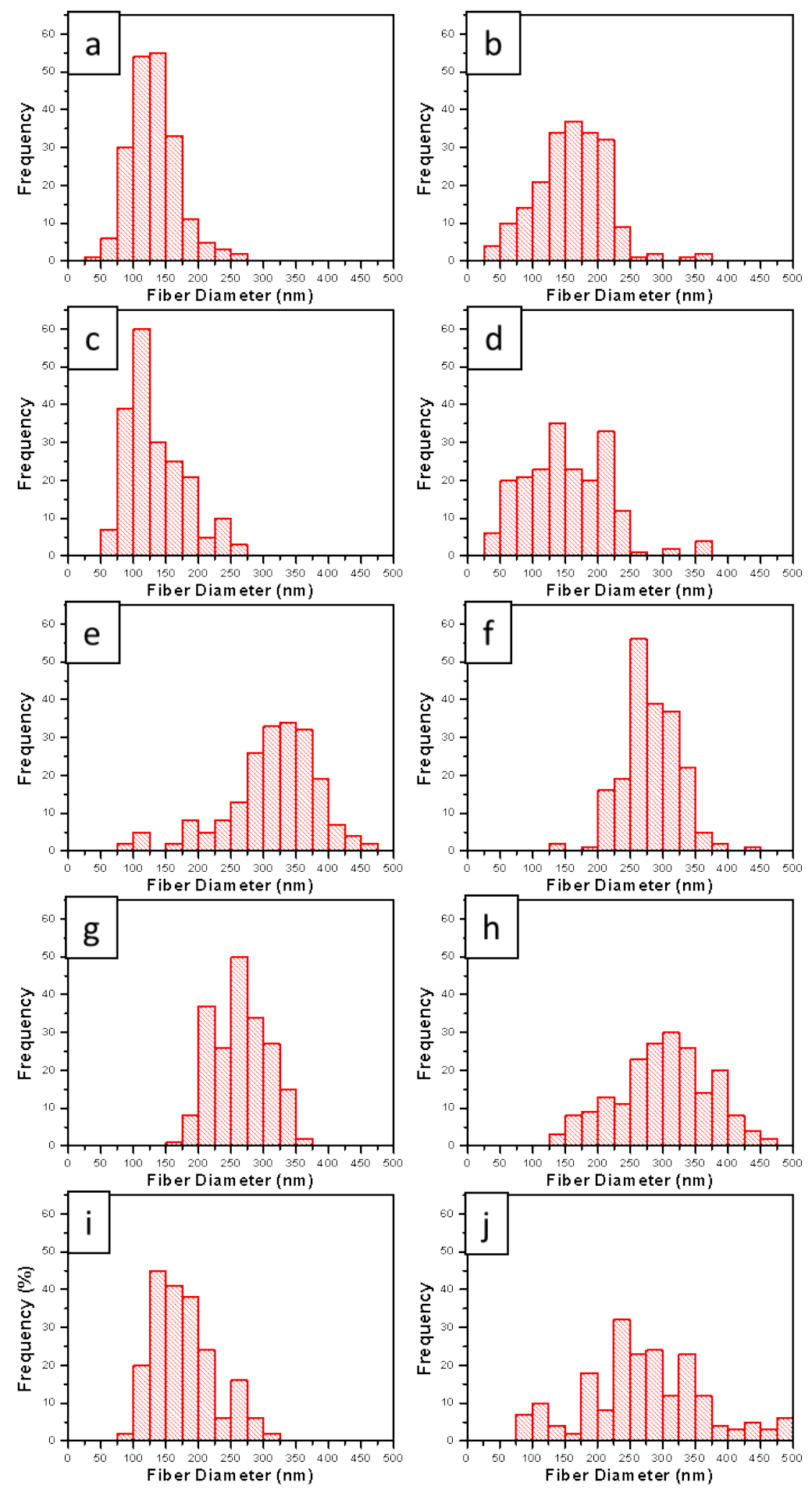

Figure S2. Electrospun nanofiber diameter histograms. (a) PEO S; (b) PVP S; (c) PEO-PANI S; (d) PVP-PANI S; (e) PEO/PVP SBS; (f) PEO-PANI/PVP-PANI SBS; (g) PVP/PEO-PANI SBS; (h) PEO/PVP-PANI SBS; (i) PEO/PEO-PANI SBS; and (j) PVP/PVP-PANI SBS. 

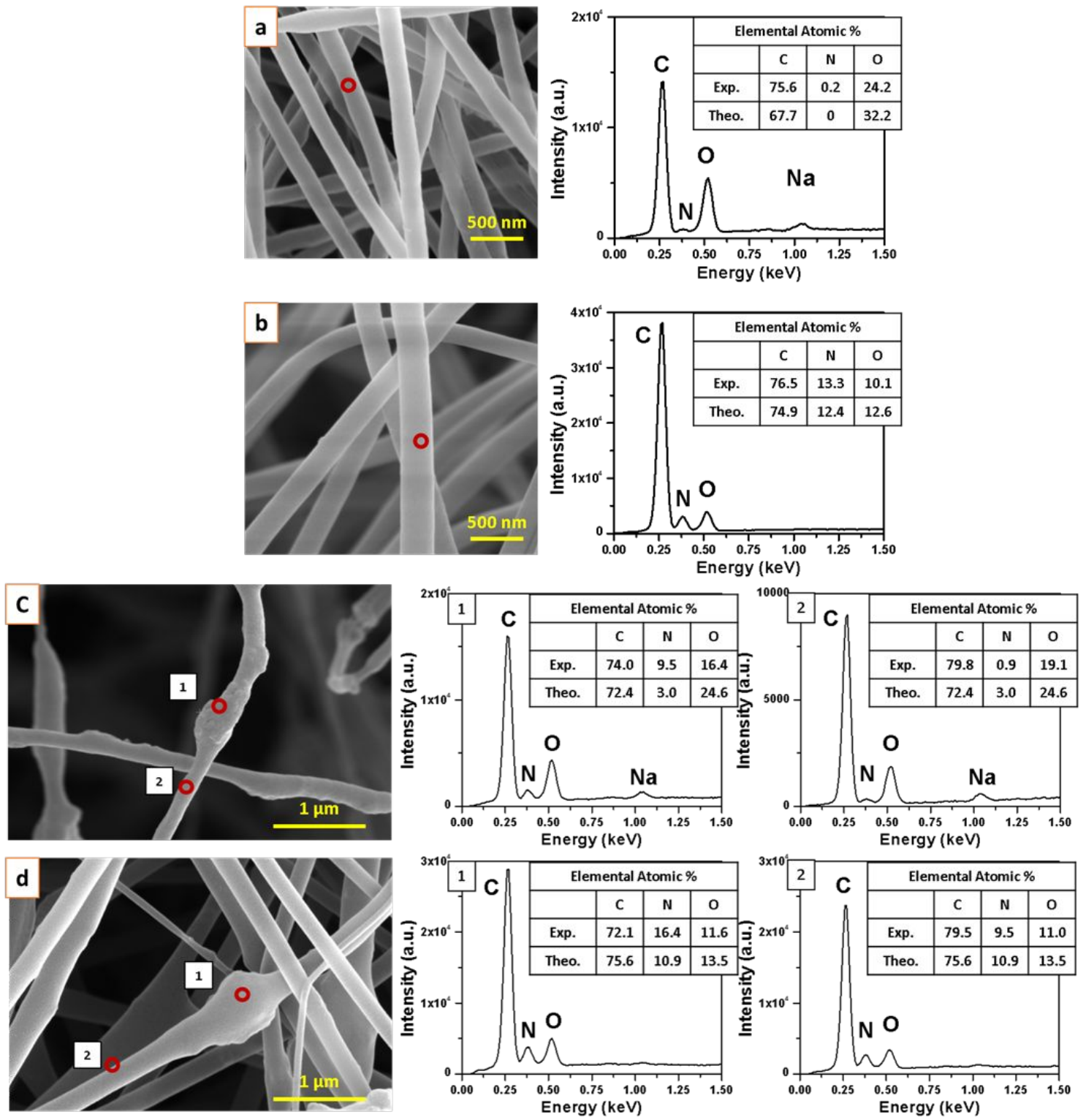

Figure S3. EDX-SEM analysis of single jet spun fibers. (a) PEO S; (b) PVP S; (c) PEO-PANI S; (d) PVP-PANI S. 


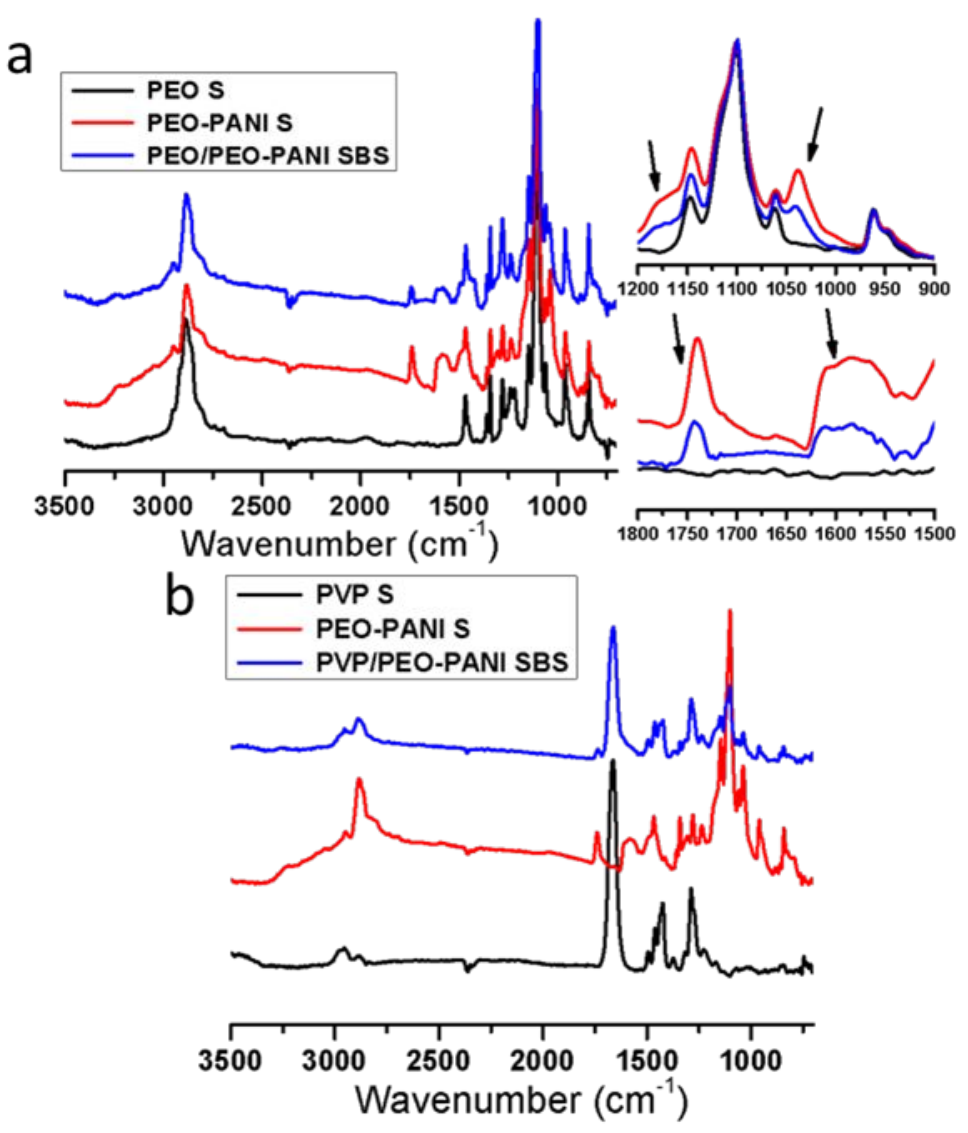

Figure S4. Representative FT-IR results of fiber mats: (a) homo-SBS PEO/PEO-PANI, PEO S, and PEO-PANI S; (b) hetero-SBS PVP/PEO-PANI, PVP S, and PEO-PANI S.

IR results of SBS fiber mats were consistent with the formulas used to prepare these fibers. In Figure S3-a, the relative amount of PEO and PANI in the three fibers could be deduced from the intensity of several signature peaks in PEO and PANI-CSA. The peak at $1105 \mathrm{~cm}^{-1}$ was assigned to C-O stretching of the ether bond in PEO. ${ }^{1}$ Peaks at $1037 \mathrm{~cm}^{-1}, 1182 \mathrm{~cm}^{-1}, 1735 \mathrm{~cm}^{-1}$ and around $1600 \mathrm{~cm}^{-1}$ were assigned to the $\mathrm{S}=\mathrm{O}$ sulfoxide stretching in CSA, absorption of the sulfonic stretch caused by doping reaction, $\mathrm{C}=\mathrm{O}$ carbonyl stretching in $\mathrm{CSA}$, and conjugated $\mathrm{C}=\mathrm{C}$ bond stretching in PANI, respectively. ${ }^{2-5}$ The PEO $\mathrm{S}$ fiber mat showed no peaks at the above-mentioned wavenumbers. When compared to the PEO-PANI S and PEO/PEO-PANI SBS, the amount of PANI-CSA in PEO-PANI S was larger than in the SBS fiber mat, which was in good agreement with the expected level.

In Figure S3-b, the two feature peaks that indicated the relative amount of PVP and PEO were $1655 \mathrm{~cm}^{-1}$ that was resulted from the stretching of carbonyl bond on PVP backbones, ${ }^{6}$ and 1105 $\mathrm{cm}^{-1}$ that was due to the asymmetric stretching of the ether bond on PEO backbones as mentioned above. In the PVP/PEO-PANI SBS fibers, the amount of PVP was much more than the amount of PEO, which was consistent with the theoretical concentration of PVP in the whole SBS fibers, i.e., $62.5 \%$. 


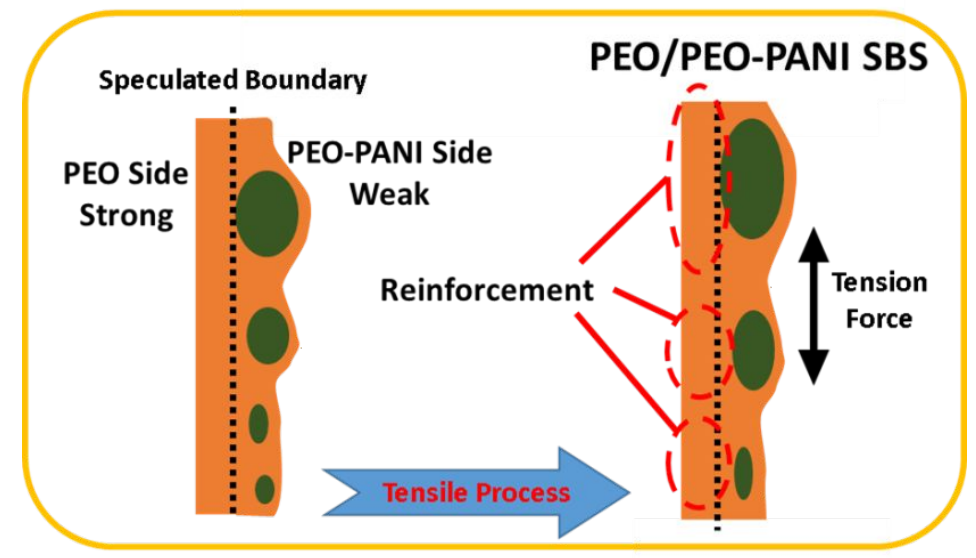

Scheme S1. Conceptual model illustrating nanofiber tensile performance: PEO/PEO-PANI SBS.

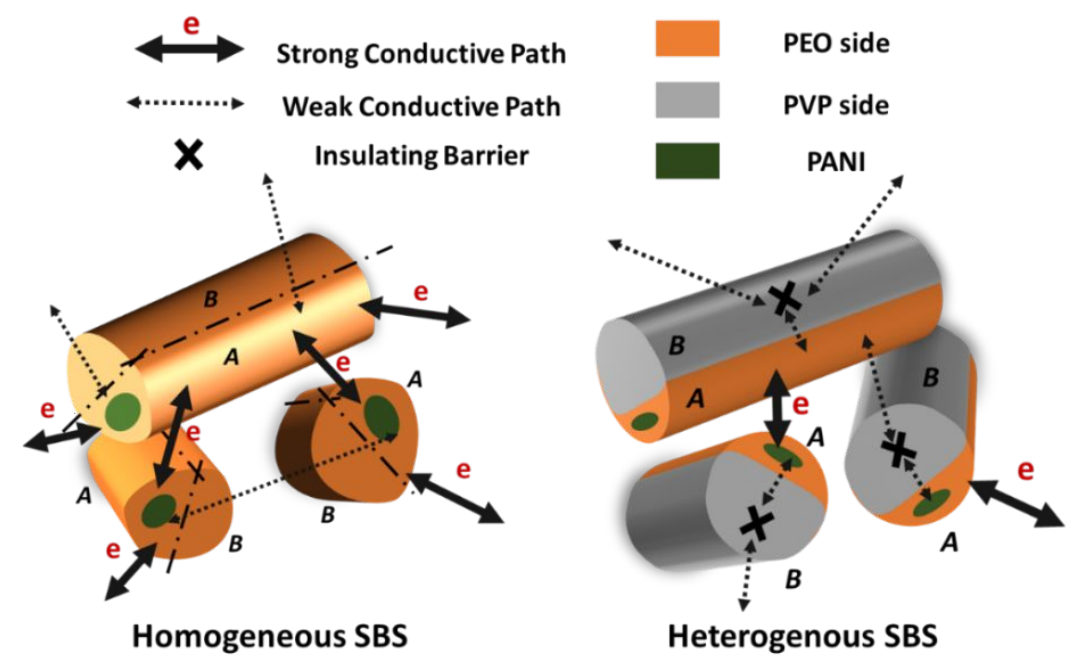

Scheme S2. Speculated model of conducting paths in SBS fiber mats using PEO/PEO-PANI SBS and PVP/PEO-PANI SBS as examples for homo- and hetero-SBSs. 

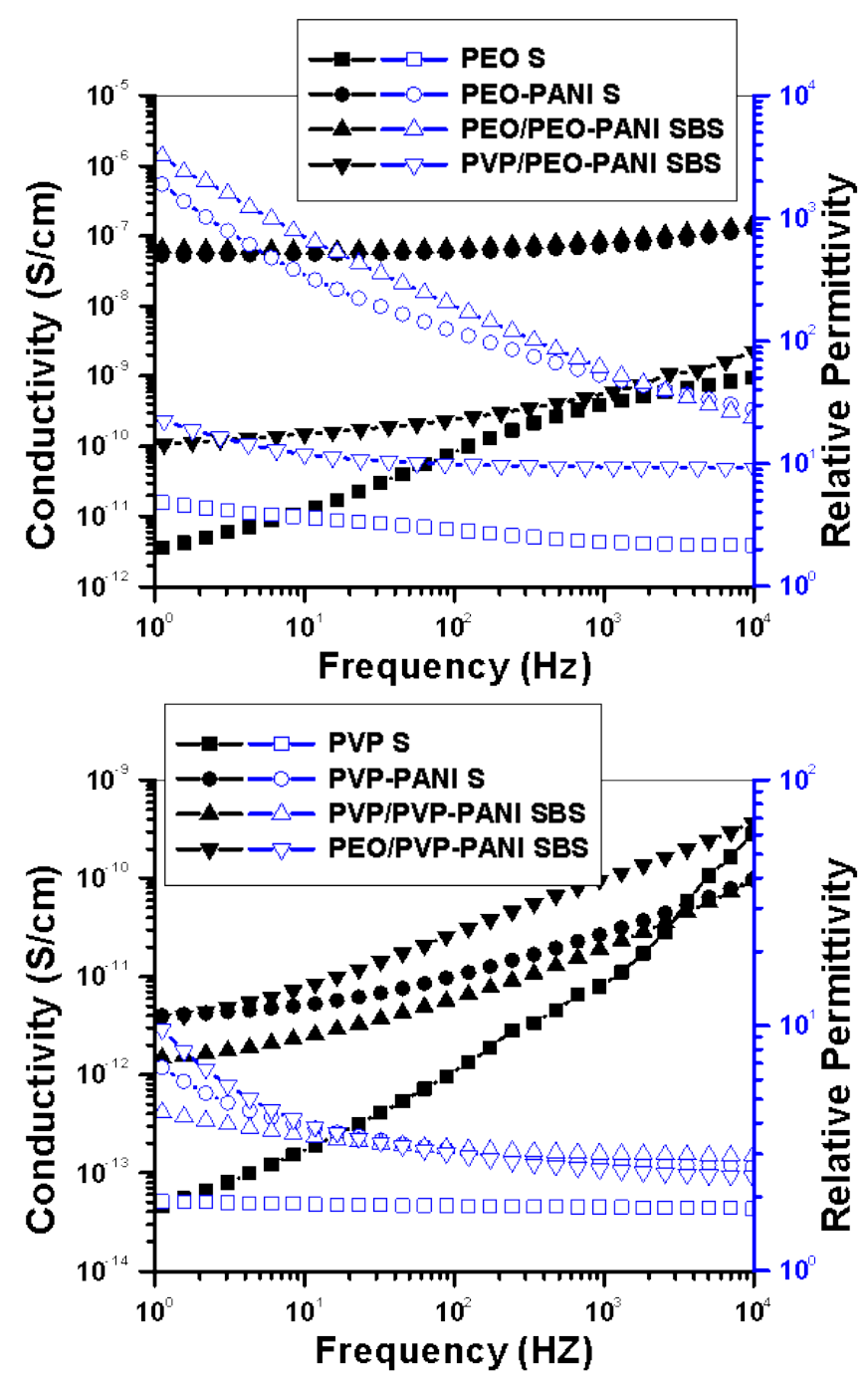

Figure S5. Electrical conductivity and relative permittivity $\left(\varepsilon_{\mathrm{r}}\right)$ of fiber mats.

The matrix polymers, PEO and PVP, both had very low relative dielectric permittivity $\left(\mathcal{E}_{\mathrm{r}}\right)$, which was as small as 2 under frequency of $10^{4}$ to $10^{\circ} \mathrm{Hz}$. $\varepsilon_{\mathrm{r}}$ for PEO was slightly frequency-dependent and that of PVP was almost constant. The addition of PANI caused the $\varepsilon_{\mathrm{r}}$ of both PEO-PANI S and PEO/PEO-PANI homoSBS to increase significantly at lower frequency. Under $50 \mathrm{~Hz}$, compared to PEO S, the $\varepsilon_{\mathrm{r}}$ of PEO/PEOPANI SBS increased from approximately 2.5 to 206.5, which was slightly higher than that of PEO-PANI $\mathrm{S}(\sim 138.7)$. This $\varepsilon_{\mathrm{r}}$ made the PEO-PANI S and PEO/PEO-PANI SBS mats very promising materials for actuators under medium-low frequency. In contrast, the $\varepsilon_{\mathrm{r}}$ of PVP was not significantly enhanced by PANI, and they were less frequency-dependent. For instance, at $50 \mathrm{~Hz}$, the $\varepsilon_{\mathrm{r}}$ of PVP S, PVP-PANI S, and PVP/PVP-PANI SBS were 1.8, 2.1, and 2.1, respectively. 


\section{References}

1. Caykara, T.; Demirci, S.; Eroğlu, M. S.; Güven, O. Poly (ethylene oxide) and its blends with sodium alginate. Polymer 2005, 46 (24), 10750-10757.

2. Raut, B. T.; Chougule, M. A.; Nalage, S. R.; Dalavi, D. S.; Mali, S.; Patil, P. S.; Patil, V. B. CSA doped polyaniline/CdS organic-inorganic nanohybrid: physical and gas sensing properties. Ceramics International 2012, 38 (7), 5501-5506.

3. Raut, B. T.; Chougule, M. A.; Ghanwat, A. A.; Pawar, R. C.; Lee, C. S.; Patil, V. B. Polyaniline-CdS nanocomposites: effect of camphor sulfonic acid doping on structural, microstructural, optical and electrical properties. Journal of Materials Science: Materials in Electronics 2012, 23 (12), 2104-2109.

4. Lee, H. T.; Wang, C. C. Effects of interactions among polyaniline, camphorsulfonic acid and silica on the structure and properties of their conductive hybrids. Polymer Engineering \& Science 2008, 48 (3), 439-447.

5. Bhadra, J.; Madi, N. K.; Al-Thani, N. J.; Al-Maadeed, M. A. Polyaniline/polyvinyl alcohol blends: Effect of sulfonic acid dopants on microstructural, optical, thermal and electrical properties. Synthetic Metals 2014, 191, 126-134.

6. Baykal, A.; Bıtrak, N.; Ünal, B.; Kavas, H.; Durmus, Z.; Özden, Ş.; Toprak, M. S. Polyol synthesis of (polyvinylpyrrolidone) PVP-Mn3O4 nanocomposite. Journal of alloys and compounds 2010, 502 (1), 199-205. 